\title{
Fast Opening Gas Valve for the TCABR Pellet Injector
}

\author{
Alvaro Vannucci, Carlos Mariz de O. Teixeira, Fernanda Sá Teixeira, José H. Vuolo, \\ Alexandre C. de Paulo*, Fernando Horita, Francisco T. Degasperi*, Juan I. Elizondo, \\ Edson K. Sanada, Vittorio A.L. Almeida, and Fábio Pantano \\ Instituto de Física, Universidade de São Paulo, 05508-900, São Paulo, SP, Brasil \\ *Faculdade de Tecnologia de São Paulo, FATEC, 01124-060, São Paulo, SP, Brasil
}

Received on 03 February, 2004; revised version received on 04 June, 2004

\begin{abstract}
Fast acting gas valves that operate between two very different pressure environments, which may differ in several orders of magnitude, are not available commercially and, therefore, they must be specifically projected and constructed. For the TCABR impurity pellet injector, under construction, an electromagnetic valve has been built which operates for gas pressure that ranges from 20 to $40 \mathrm{~atm}$ at one side of the valve to $10^{-9} \mathrm{~atm}$ at the other side. The working principle of the valve is based on the displacement of an aluminum disk (which controls the gas flux) from its closing position by an electromagnetic force, in result to an induced electric current on the disk caused by a magnetic flux variation. Experimental results showed that the electromagnetic impulse on the disk lasts for $200 \mu s$ and takes less than $30 \mathrm{~ms}$ for the valve to shut up again.
\end{abstract}

\section{Introduction}

Fast and controlled gas injection can be considered as an useful technique in several and different areas of experimental physics research. In particular, for the magnetic confinement of plasmas, devices like tokamaks are generally employed in which the use of fast and controlled gas injection is required to operate properly. In this paper, in particular, the construction details are given of a gas injection system that will be used in a pellet injector for the TCABR tokamak, at the Physics Institute of the University of São Paulo.

As already have been observed previously in other tokamaks, the interaction of the plasma with impurity pellets (boron, carbon, aluminum, tungsten, etc.) provokes a local and sudden increase of the concentration of impurity ions, leading to a strong dissipation of the stored energy and, many times, to a modification of the plasma equilibrium and stability [1-4].

In JET, for instance, it has been observed that the use of solid D2 pellets led to the creation of a structure similar to a snake in the profiles of the softray-X emission. From this observation it was possible to determine, in the plasma, the exact location of the $\mathrm{q}=1$ magnetic surface [2].

In the tokamak ASDEX, on the other hand, neon pellets were injected with the objective of controlling and mitigating the disruptive instabilities. In that work, however, since there were no possibility of foreseeing when the disruptions would take place, the neon pellets were shot during the plasma discharges without the concern of whether the disruption has already been triggered or not. It could be verified that the injected pellets irradiated up to $90 \%$ of the total plasma stored energy in time intervals less than $1 \mathrm{~ms}$ [3]. Furthermore, it was demonstrated that the mechanical stresses induced in the whole tokamak structure, due to the abrupt ending of the plasma confinement, were reduced in $50 \%$, on average, when the neon pellets were injected.

Now, in the MT-1M and TEXTOR-94 tokamaks, the use of aluminum micro-pellets made possible a direct analysis of the plasma-pellets interaction, which typically formed light emitting grooves [4]. The experimental results obtained could be properly explained in terms of the aluminum sublimation rate, that was calculated from the expansion of the vaporized aluminum cloud.

For the TCABR tokamak, an impurity pellet injector is now under construction with the objective of investigating the transport of particles in different discharge conditions, and also of studying how this system could be used to control the triggering process of disruptive instabilities. The most sensitive part of the project is related to the construction of a fast acting electromagnetic gas valve, and the corresponding high current (up to $6 k A$ ) and high voltage (up to $7 \mathrm{kV}$ ) power supply. The details of the project and construction of these components are given and fully discussed in this article.

\section{Electromagnetic valve}

Fast opening and closing gas valves have innumeous application in experimental physics. In plasma research, since the beginning many works have been carried out using these special valves, with different working concepts [5-7]. The principle of operation of the fast acting valve that was built for the TCABR pellet injector is based on Foucault currents that are induced in an aluminum disk (diameter $d=2.6 \mathrm{~cm}$ and mass $m=2.3 \mathrm{~g}$ ), seated on a magnetic coil with low inductance $(3.0 \mu \mathrm{H})$ and low electric resistance $(0.98 \Omega)$ in 


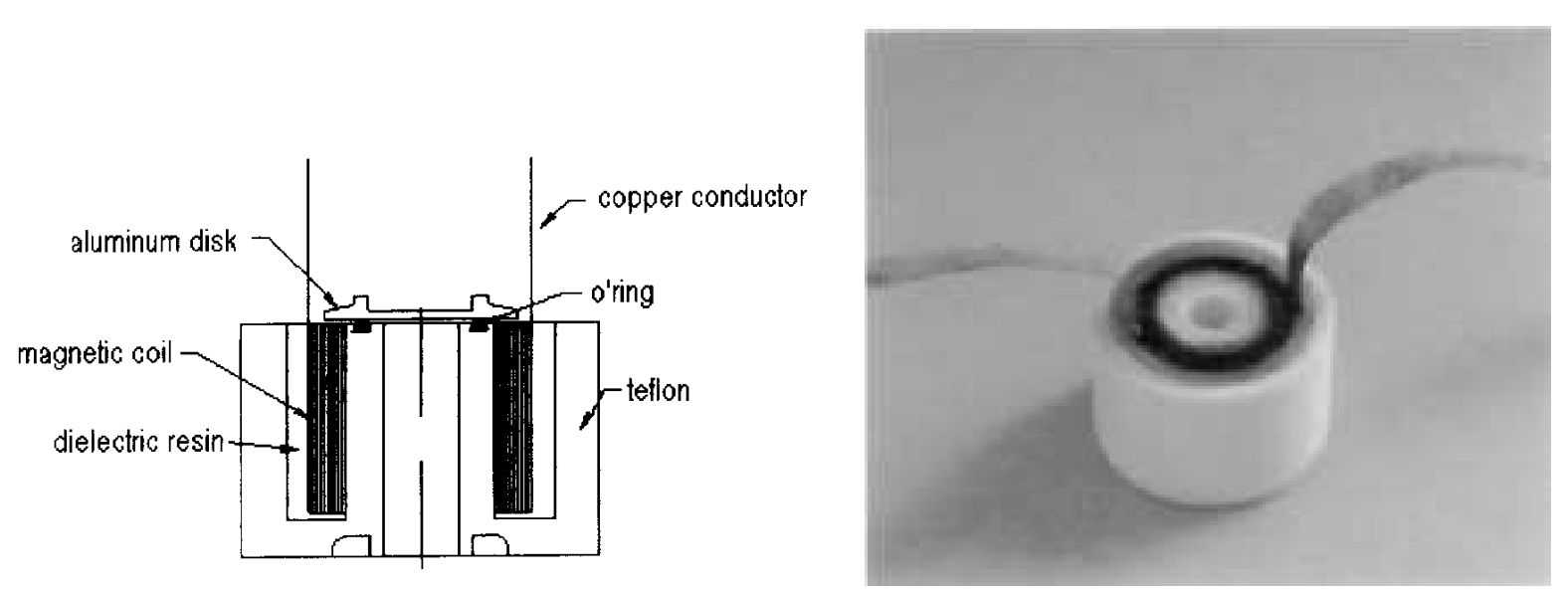

Figure 1. Magnetic coil constructed for the TCABR pellet injector: (a) schematic drawing and (b) the valve in its final form.
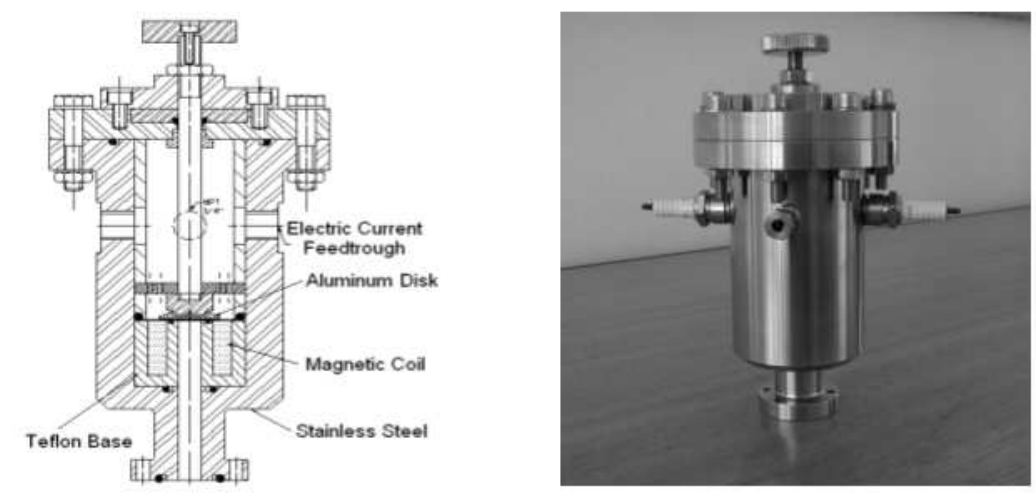

Figure 2. High pressure gas chamber constructed for the TCABR pellet injector: (a) schematic drawing and (b) the chamber in its final form.

which up to $6 k A$ currents are produced. The construction of the magnetic coil was accomplished by winding a copper ribbon (length $L=1 \mathrm{~m}$, width $w=8 \mathrm{~mm}$ and thickness $t=0.1 \mathrm{~mm}$ ), totalizing 12 turns. A good electric insulation between each winding was obtained using a continuous mylar ribbon (width $h=20 \mathrm{~mm}$ and thickness $z=0.3 \mathrm{~mm}$ ) and, afterwards, dipping the whole assembly in a liquid epoxy resin that, after the hardening, gave body to the reel and also served as element of mechanical fixation of the reel to a teflon base (Fig. 1).

The coil parameters, mentioned above, were chosen as to produce a mechanical force on the aluminum disk against the high gas pressure, of the order of $1100 \mathrm{~N}$ or greater, to allow a determined amount of gas to pass through the valve (this will happen when the disk, that is seated on the O'ring of the valve is moved from its resting position). This force is given by

$$
m . a=F_{e}-P-F_{p}-F_{m}
$$

where $F_{e}$ is the induced electromagnetic force, $F_{P}$ is the force (pressure) that the gas exercises on the disk, $P$ represents the weight of the disk and $F_{m}$ the force exercised by a spring that is located above the disk, which helps the disk to come back to its original position.

Simple calculations for gas pressures between $20-40$ atm show that the necessary electromagnetic force to the ring is obtained for electric currents $I \sim(5 \pm 1) \mathrm{kA}$ flowing through the coil windings. For the TCABR pellet injector, these currents are obtained when voltages $V \sim(5 \pm 1) \mathrm{kV}$ are applied to the terminals of the magnetic coil.

\section{High pressure gas chamber}

Figure 2 shows the high pressure gas chamber in which the fast opening valve was installed. This chamber, with cylindrical geometry, was projected and constructed using $316 \mathrm{~L}$ stainless steel. To control the amount of gas passing through the valve which is proportional to the period of time the aluminum disk takes to return to its resting position, an adjusting rod was positioned inside the chamber. Thus, the displacement of the disk can be easily controlled by simply adjusting the length of the rod.

The chamber was initially projected assuming that the electromagnetic valve placed inside would be electrically 


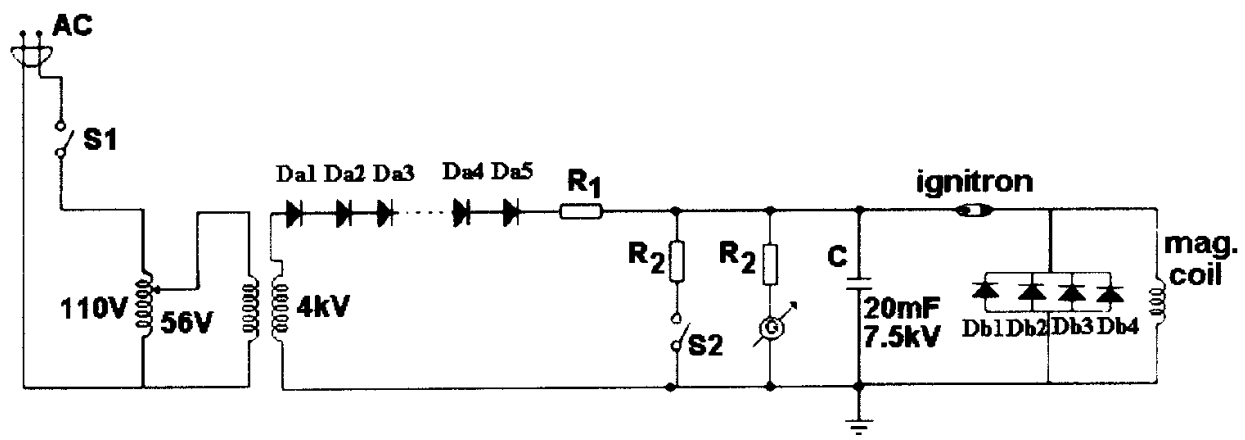

Figure 3. Schematic of the TCABR pellet injector electric circuit.

connected to the high voltage and high current power supply through two regular automotive spark plugs. However, when tested, the commercial spark plugs did not function correctly as feed-throughs, and they had to be rebuilt altogether, using more robust materials and choosing a more adequate assembly. Teflon was used as insulator.

\section{Power supply}

The fast acting valves high current and high voltage power supply was constructed, basically, using diodes, a transformer and a capacitor which was connected to the electric circuit through an ignitron switch (Fig. 3). The power supply was projected as to permit different voltage values to be applied to the transformer through a variac, at the proportion of 90:6400. The secondary of the transformer is connected to the charging circuit which is constituted by 16 rectifying 1N4007 diodes (reverse voltage $V_{R}=1 \mathrm{kV}$, direct current $I_{D}=1 A$ - connected in series $)$, a resistor $\left(R_{1}=100 \mathrm{k} \Omega\right)$, to limit the charging current to $50 \mathrm{~mA}$, and the capacitor $\left(C=20 \mathrm{mF}, V_{C}=7.5 \mathrm{kV}\right)$. The valves firing circuit is mainly composed by the ignitron, 4 high current KHP10 diodes (reverse voltage $\mathrm{VR}=10 \mathrm{kV}$, direct peak current $I_{D P}=2.5 \mathrm{kA}$ - connected in parallel) and the encapsulated magnetic coil. When in operation, the voltage used to fire the valve should not exceed the limit of $6.5=7.0 \mathrm{kV}$, to prevent the possibility of overloading the capacitor. To measure the electric current flowing through the valves magnetic coil, a Rogowski coil was specifically built, and properly calibrated, for this project.

\section{System response}

To test how the system responds to a capacitor discharge, the constructed Rogowski coil and an integrator, coupled to an oscilloscope, were used to measure the electric current flowing in the coil. In Fig. 4 the temporal current profile obtained when a voltage of $4 k \mathrm{~V}$ is applied to the coil is shown. The current duration is typically $\tau_{d} \sim 200 \mu \mathrm{s}$ and the peak of the current, in this case, is $I_{P} \sim 4.6 k A$. During the tests it was observed that the force on the aluminum disk varied considerably in relation to the existing distance between the disk and the top surface of the magnetic coil. The distance $d=0.5 \mathrm{~mm}$ was observed to be the one that yielded the best results without short-circuiting the coil windings during the discharges. The intensity of this force can be calculated according to the expression [7]:

$$
F_{e}=\frac{\pi \mu n^{2}}{5500} \frac{R^{2}}{d^{2}} I^{2}
$$

where $\mu$ is the permeability of the medium (polyester resin), $n$ is the number of coil windings, $R$ is the coil radius and $I$ is the current in the windings.

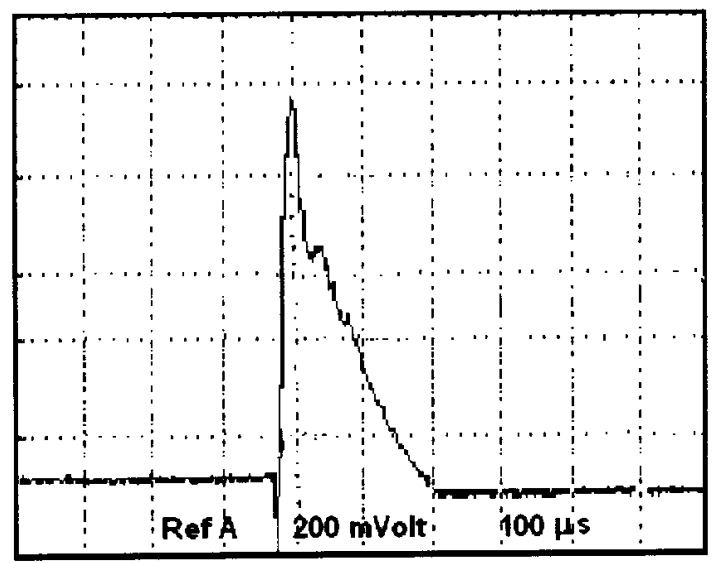

Figure 4. Typical electric current signal in the coil windings.

Finally, an experimental analysis was carried out to evaluate the time the valve remains opened due to a capacitor discharge. The force exercised on the valves aluminum disk due to the gas pressure inside the camera was simulated using a set of small brass weights, of standardized masses, placed over the metallic disk, as shown in Fig. 5. When the disk lifts up the weights, a laser beam hits the light sensor placed at the other side of the arrangement, inducing a signal which is measured by an oscilloscope. After the weights return to the initial position, the laser beam is blocked and no signal is measured anymore by the oscilloscope. As shown in Fig. 6 the valve remains opened for about $30 \mathrm{~ms}$ when a voltage $V=4.5 \mathrm{kV}$ is applied. Under normal operational conditions, the valve aperture time can be controlled at will by controlling the voltage on the coil terminals and, therefore, the current in the windings. 


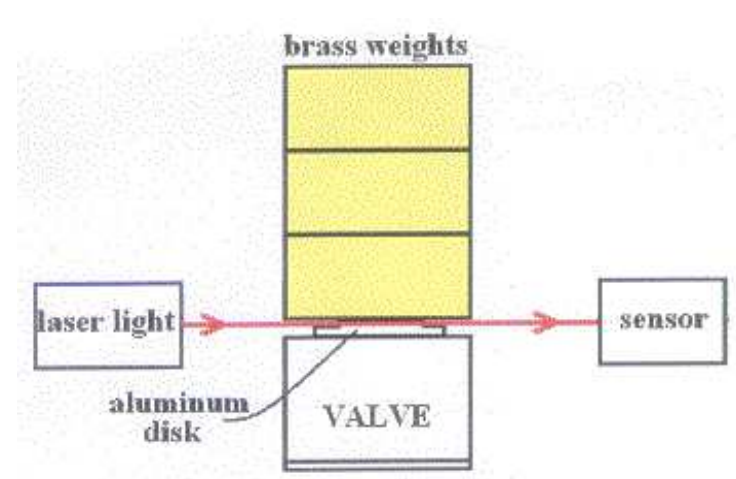

Figure 5. Experimental arrangement idealized to evaluate the valves opening time.

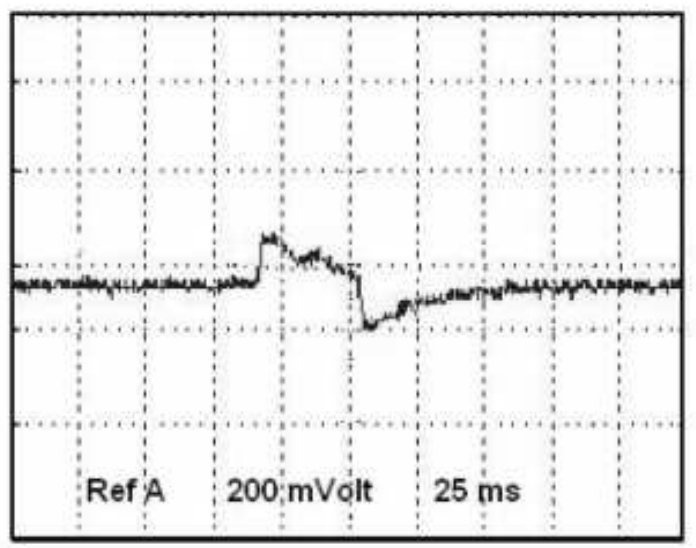

Figure 6. Signal obtained using the set-up shown in Figure 5 for a voltage of $4.5 \mathrm{kV}$.

\section{Conclusion}

A fast opening electromagnetic gas valve was built and will be used in the TCABR pellet injector, presently under construction. The valve was projected as to operate under pressures that range from 20 to $40 \mathrm{~atm}$ at one side of the valve to $10^{-9}$ atm at the other side. The working principle of the valve is based on the displacement of an aluminum disk (which controls the gas flux) from its closing position by an electromagnetic force, in result to an induced electric current on the disk caused by a magnetic flux variation. Experimental results showed that the electromagnetic impulse on the disk lasts for $200 \mu \mathrm{s}$ and takes about $30 \mathrm{~ms}$ for the valve to shut up again.

\section{Acknowledgments}

The authors want to thank FAPESP - Fundação de Amparo à Pesquisa do Estado de São Paulo for the financial support.

\section{References}

[1] S.L. Milora et al. Nucl. Fusion 35 , 657 (1995).

[2] A. Weeler, A. D. Cheetham, A.W. Edwards et al Phys. Rev. Lett. 59, 2303 (1987).

[3] G. Pautasso, K. Bluchl, J.C. Fuchs, et al. Nucl. Fusion 36, 1291 (1996).

[4] G. Kocsis et. al Plasma Phys.Control. Fusion 41, 881 (1999).

[5] B. Gorowitz, K. Moses, and P. Gloersen, Rev. Sci. Instrum. 31, 146 (1960).

[6] R.S. Lowder, F.C. Hoh, Rev. Sci. Instrum. 33, 1236 (1962).

[7] B. Novak, S.Pekarek, Rev. Sci. Instrum. 41, 369 (1970). 